\title{
Splitting Inductance Tuning Method to Eliminate High Frequency Oscillation in Dual Active Bridge Converter
}

Wang, Chang; Huang, Jiasheng; Zsurzsan, Gabriel; Zhang, Zhe

Published in:

Proceedings of 2021 IEEE Energy Conversion Congress and Exposition

Link to article, DOI:

10.1109/ECCE47101.2021.9595627

Publication date:

2021

Document Version

Peer reviewed version

Link back to DTU Orbit

Citation (APA):

Wang, C., Huang, J., Zsurzsan, G., \& Zhang, Z. (2021). Splitting Inductance Tuning Method to Eliminate High Frequency Oscillation in Dual Active Bridge Converter. In Proceedings of 2021 IEEE Energy Conversion Congress and Exposition (pp. 2930-2934). IEEE. https://doi.org/10.1109/ECCE47101.2021.9595627

\section{General rights}

Copyright and moral rights for the publications made accessible in the public portal are retained by the authors and/or other copyright owners and it is a condition of accessing publications that users recognise and abide by the legal requirements associated with these rights.

- Users may download and print one copy of any publication from the public portal for the purpose of private study or research.

- You may not further distribute the material or use it for any profit-making activity or commercial gain

- You may freely distribute the URL identifying the publication in the public portal 


\section{Splitting Inductance Tuning Method to Eliminate High Frequency Oscillation in Dual Active Bridge Converter}

\author{
Chang Wang \\ DTU Electrical Engineering \\ Technical University of Denmark \\ Kongens Lyngby, Denmark \\ chawa@elektro.dtu.dk \\ Zhe Zhang \\ DTU Electrical Engineering \\ Technical University of Denmark \\ Kongens Lyngby, Denmark \\ zz@elektro.dtu.dk
}

\author{
Jiasheng Huang \\ DTU Electrical Engineering \\ Technical University of Denmark \\ Kongens Lyngby, Denmark \\ jiahuan@elektro.dtu.dk
}

\author{
Gabriel Zsurzsan \\ DTU Electrical Engineering \\ Technical University of Denmark \\ Kongens Lyngby, Denmark \\ tgzsur@elektro.dtu.dk
}

\begin{abstract}
There is a growing trend towards operating higher frequency in dual active bridge (DAB) converters to achieve higher power density. At the meantime, the high frequency oscillation (HFO) problem introduced by the parasitic components of the transformer and high $d v / d t$ of the ac link excitation voltage becomes detrimental. Matching the oscillation frequency with the zero crossing points (ZCP) of the DAB $d v / d t$ excitation has been proven effective to eliminate the HFO problem. In this paper, a splitting inductance tuning method is proposed to adjust the circuit frequency characteristic and place the lowest impedance path at the ZCP frequency, thus eliminating the HFO. Theoretical analysis is given along with the experimental results from a $1 \mathrm{MHz}$ Gallium Nitride (GaN) based DAB converter prototype.
\end{abstract}

Keywords-Dual active bridge, high frequency oscillation, splitting inductance tuning method, gallium nitride

\section{INTRODUCTION}

There is a growing trend to utilize the dual active bridge (DAB) converters into industrial application since its low component stress, bidirectional power flow capability, buck/boost operation mode, large power capability and ease of realizing soft switching [1][2]. The DAB converter is widely employed in electric vehicles, fuel cell power conversion, renewable energy storage system, etc [3][4]. With the desire for higher efficiency and higher power density of power converters, the operating frequency is usually increased to mega-hertz $(\mathrm{MHz})$ range [5][6]. On the one hand, the increase of frequency brings to the decrease of the passive components' size due to Faraday's law. On the other hand, the trace inductance or parasitic capacitance in the circuit becomes non-negligible, which sometimes can cause failure of the converters' operation if not properly considered. With high operating frequency, the transformer's parasitic capacitances become non-negligible in DAB converter [7][8]. With high $d v / d t$ ratio of the ac link voltage, it introduces the high frequency oscillation (HFO) to the circuit as shown in Fig.1. This is due to the ac link voltage excitation contains large amounts of harmonics, while the parasitic capacitances of the $\mathrm{DAB}$ converter provide a certain frequency harmonic path together with the transformer and inductances of the circuit. In previous literature [9], a parallel capacitor has been added to the drain-to-source of the MOSFET to increase the rising time of the ac link voltage. This is aimed to decrease the overall frequency of the harmonic spectrum. However, this also decreases the soft-switching speed due to the extra capacitor and brings in extra loss. Which is not suitable for high frequency operation when the soft-switching and its speed are vital to the converters' overall performance. To solve the HFO problem with high frequency operation, the approach of distributing the external inductance on both sides of the transformer is proposed in this paper. The tuning of circuit impedance and frequency characteristic is elaborated. A $1 \mathrm{MHz}$ GaN-based DAB experimental prototype is built and tested to validate the theoretical analysis. The experimental comparison has been made to validate the proposed method.

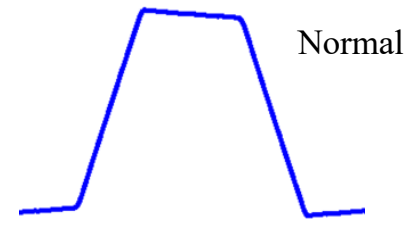

(a)

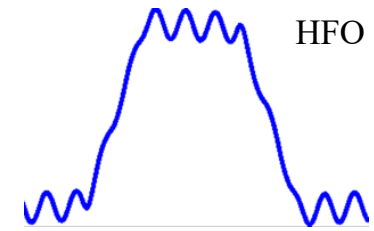

(b)
Fig.1. Inductor Current Waveform: (a) Typical Trapezoidal Waveform (b) Waveform with High Frequency Oscillation (HFO)

\section{DAB IMPEDANCE NETWORK}

The topology of the DAB converter including the transformer parasitic capacitors $C_{\mathrm{p} 1}$ and $C_{\mathrm{p} 2}$ is shown in Fig.2. The $L_{\mathrm{k} 1}$ and $L_{\mathrm{k} 2}$ represent the leakage inductances of the transformer while the $L_{\mathrm{e} 1}$ and $L_{\mathrm{e} 2}$ are the external inductances splitting into the high-voltage (HV) side and low-voltage (LV) side of the transformer. To keep the equivalent inductance referred to the primary side of the transformer $L_{\mathrm{e}}$ unchanged, the two external inductances have the following relation,

$$
L_{e 1}+N^{2} L_{e 2}=L_{e}
$$

Throughout theoretical analysis and simulations in [10], it is revealed that the existence of $C_{\mathrm{p} 1}$ and $C_{\mathrm{p} 2}$ leads to a nonsinusoidal switching-node voltage $V_{\text {acc }}$. The increase of the high-voltage side external inductance $L_{\mathrm{e} 1}$ forms a quasisinusoidal $V_{\text {acl }}$ which further reduces the minimum dead time to achieve ZVS. The specification and constructed experimental prototype are listed in TABLE I, the parameters can be extracted as shown in TABLE II. During the transient 
period when the $S_{1}$ and $S_{4}$ turned on, $S_{2}$ and $S_{3}$ turned off, the interfacing points $V_{\text {ac1 }}$ rises from $-U_{\text {in }}$ to $U_{\text {in. }}$ The high frequency excitation voltage is applied on the impedance network seen from $V_{\text {ac1 }}$. The equivalent impedance network is shown in Fig.3. and can be expressed mathematically as $Z_{\text {in. }}$ It can be derived from the bode plot of $Z_{\text {in }}$ that there are two zeros of the impedance network and the first zero is where the low impedance path exists. Thus, the harmonics of the same frequency can pass through the impedance network and cause distortion.

$$
\begin{gathered}
Z_{s}=j \omega L_{k 2}+\frac{\frac{j \omega L_{e 2}+R_{e q}}{j \omega C_{p 2}}}{j \omega L_{e 2}+R_{e q}+\frac{1}{j \omega C_{p 2}}} \\
Z_{s e q}=\frac{N^{2} Z_{s} \cdot j \omega L_{M}}{N^{2} Z_{s}+j \omega L_{M}} \\
Z_{i n}=j \omega L_{e 1}+\frac{\frac{j \omega L_{k 1}+Z_{s e q}}{j \omega C_{p 1}}}{j \omega L_{k 1}+Z_{s e q}+\frac{1}{j \omega C_{p 1}}}
\end{gathered}
$$

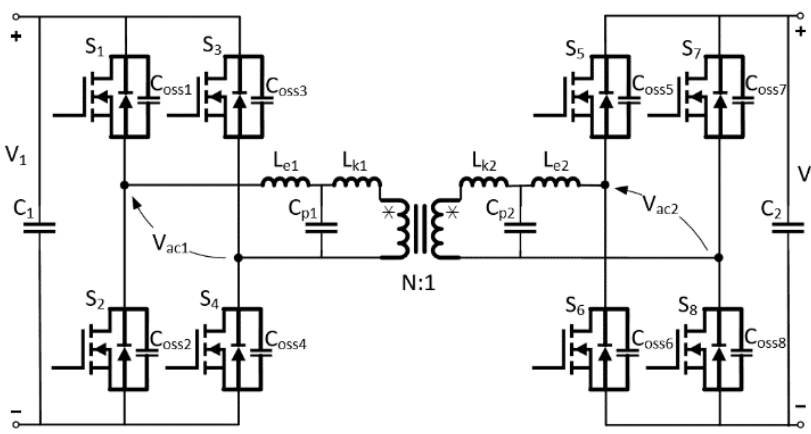

Fig.2. Topology of DAB Converter

TABLE I. Specifications of the DAB Prototype

\begin{tabular}{|c|c|c|}
\hline \multirow{4}{*}{ Specifications } & Description & Parameter \\
\cline { 2 - 3 } & $\begin{array}{c}\text { Switching frequency } \\
\text { DC voltage on the HV } \\
\text { side }\end{array}$ & $1 \mathrm{MHz}$ \\
\cline { 2 - 3 } & $\begin{array}{c}\text { DC voltage on the LV } \\
\text { side }\end{array}$ & $200 \mathrm{~V}$ \\
\cline { 2 - 3 } Power & Rated output power & $250 \mathrm{~V}$ \\
\hline \multirow{2}{*}{$\begin{array}{c}\text { semiconductor } \\
\text { Transformer }\end{array}$} & $\begin{array}{c}\text { Switches on the HV side } \\
\text { Core's material, type, size } \\
\text { and turns ratio }\end{array}$ & $\begin{array}{c}\text { GLS66502B } \\
\text { ML91S, 2×EE- } \\
21.8-11.4-15.8, \\
8: 2\end{array}$ \\
\hline
\end{tabular}

TABLE II. Measure parameters Used in Calculation and Simulation

\begin{tabular}{|l|l||l|l|}
\hline$L_{M}$ & $646 \mu \mathrm{H}$ & $N$ & 4 \\
\hline$L_{k 1}$ & $1300 \mathrm{nH}$ & $L_{k 2}$ & $81 \mathrm{nH}$ \\
\hline$C_{p 1}$ & $28 \mathrm{pF}$ & $C_{p 2}$ & $430 \mathrm{pF}$ \\
\hline$L_{e 1}$ & $0 \mu \mathrm{H}-24 \mu \mathrm{H}$ & $L_{e 2}$ & $\left(24 \mu \mathrm{H}-L_{e 1}\right) / N^{2}$ \\
\hline
\end{tabular}

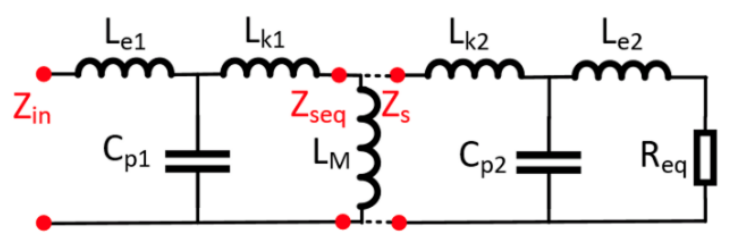

Fig.3. Equivalent Impedance Network

\section{High Frequency Oscillation Source}

The frequency spectrum of $\mathrm{dv} / \mathrm{dt}$ excitation was analyzed in [9]. The waveform of excitation voltage at ac link $V_{\text {ac1 }}$ is shown in Fig.4 and can be expressed mathematically in time domain as,

$$
V_{a c 1}(t)=\frac{2 U_{\text {in }}}{t_{\text {rise }}}\left[t u(t)-\left(t-t_{\text {rise }}\right) u\left(t-t_{\text {rise }}\right)\right]-U_{\text {in }}
$$

where the $u(\mathrm{t})$ is a unit step function, $U_{\text {in }}$ is the input voltage of the $\mathrm{DAB}$ converter, $t_{\text {rise }}$ is the rising time. Thus, the Fourier transformation can be adopted for frequency domain analysis,

$$
V_{\text {ac } 1}(j \omega)=\frac{2 U_{\text {in }}}{\omega^{2} t_{\text {rise }}}\left[\cos \left(\omega t_{\text {rise }}\right)-1-j \sin \left(\omega t_{\text {rise }}\right)\right],(\omega>0)
$$

and the corresponding magnitude can be found and shown in Fig.5.

$\left|V_{a c 1}(j \omega)\right|=\frac{2 U_{\text {in }}}{\omega^{2} t_{\text {rise }}} \sqrt{2-2 \cos \left(\omega t_{\text {rise }}\right)},(\omega>0)$

The zero crossing points (ZCP) is defined as where the above magnitude reaches zero,

$$
\omega=\frac{n \cdot 2 \pi}{t_{\text {rise }}},\left(n \in N^{*}\right)
$$

It is obvious that there are no harmonics at the ZCP frequency inside the $d v / d t$ excitation. Thus, it's vital to match the impedance path frequency with the ZCP frequency for no oscillation design. With relatively fast switching speed (high $d v / d t$ ratio), the frequency of ZCP is relatively high. So it's easier to tune the path frequency to the first ZCP frequency in this case.

$$
f_{Z C P}=\frac{1}{t_{\text {rise }}}
$$

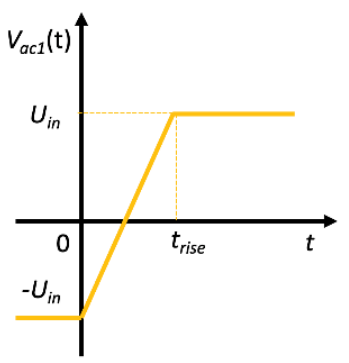

Fig.4. Excitation Voltage at ac Link 


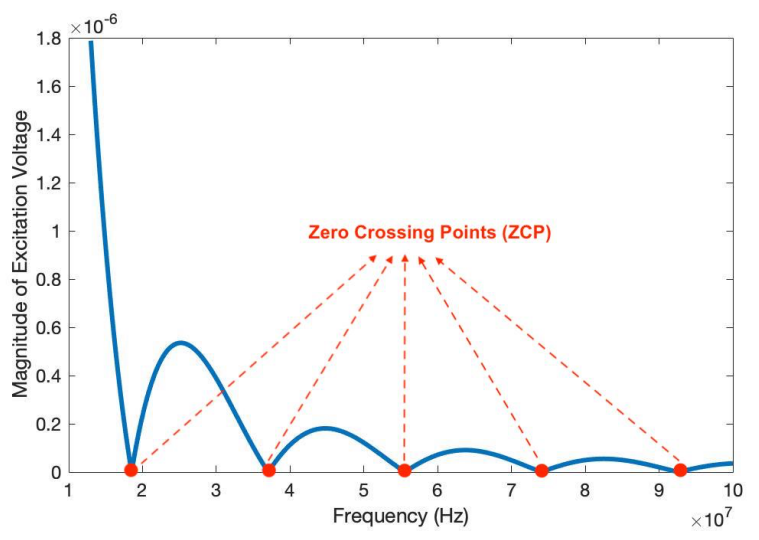

Fig.5. Excitation Voltage Frequency Spectrum

\section{Splitting Inductance Tuning Method}

With the traditional DAB converters [4] or symmetrical splitting inductance method [9], the impedance network is nonadjustable with fixed frequency response. However, this asymmetrical splitting method enables the adjustment of impedance network frequency characteristics by tuning the splitting inductance between $L_{\mathrm{e} 1}$ and $L_{\mathrm{e} 2}$. With parameters derived from the constructed prototype, the tuning frequency range of the first zero (path frequency) can be obtained by using (2) shown in Fig.6. With given ZCP frequency, corresponding inductance value $L_{\mathrm{e}}$ can be determined. Moreover, the fully ZVS range of inductance value $L_{\mathrm{e}}$ can be calculated according to [10] considering proper dead time, which is also marked as the grey area in Fig.6. It can be observed that the require $L_{\mathrm{e}}$ in this case doesn't fit for fully ZVS operation due to lack of stored energy for discharging the output capacitance of the switches. However, partial ZVS can still be achieved. Arguing that the eliminating HFO contributes more in reducing the EMI and loss while the partial ZVS of switches are acceptable, the choice of $L_{\mathrm{e}}$ mainly relies on ZCP frequency. Note that the analysis is valid for both forward and reverse conditions of bi-directional power flow. Thus, tuning the frequency of the first zero to match with the first ZCP frequency is possible within certain range.

With two sets of inductance value, one for tuning the first zero to ZCP frequency as optimal set and one for tuning the first zero away from ZCP frequency as comparing set. We may compare the simulation result as shown in Fig.7. For the optimal set, low harmonics and little current distortion can be observed comparing with other waveforms of comparing set, which is corresponding to the matching of impedance path frequency and ZCP frequency as addressed above. High frequency oscillation can be observed on the comparing set current waveform. Which also shows the existing problem and validates the splitting inductance methods.

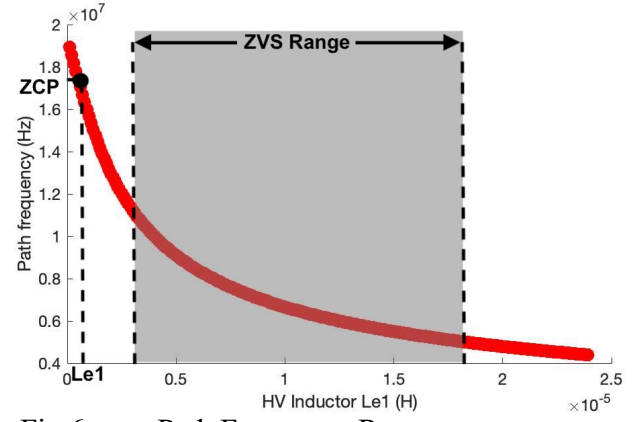

Fig.6. Path Frequency Range
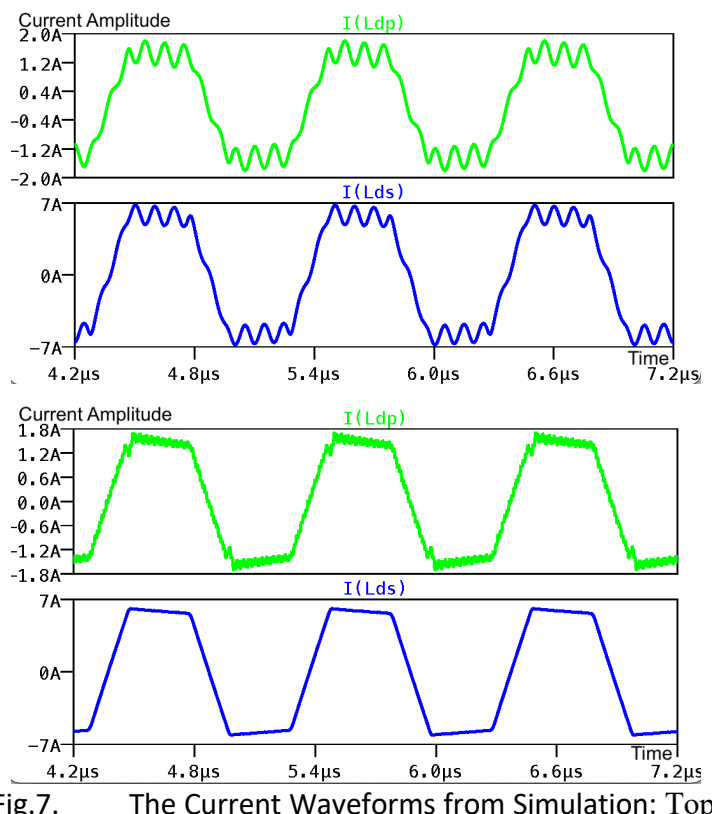

Fig.7. The Current Waveforms from Simulation: Top: Optimal Set: $L_{e 1}=10 \mu \mathrm{H} /$ Bottom: Comparing Set: $L_{e 1}$ $=0.4 \mu \mathrm{H}$

\section{EXPERIMENT}

With the constructed prototype shown in Fig.8. The waveforms are measured under the $1 \mathrm{MHz}, 200 \mathrm{~W}$ operation. The rising time trise be measured by the ac link voltage (switching node voltage) waveform shown in Fig.9. The rising time is $54.2 \mathrm{~ns}$ and the first ZCP frequency can be calculated by (7) to be $18.45 \mathrm{MHz}$. By referring to Fig.6, we can obtain the required $\mathrm{HV}$ side inductance value. The $L_{\mathrm{e} 1}=365 \mathrm{nH}, L_{\mathrm{e} 2}=$ $1.48 \mu \mathrm{H}$ are chosen as the optimal set measured by the Agilent 4294A precision impedance analyzer and the $L_{\mathrm{e} 1}=9.9 \mu \mathrm{H}, L_{\mathrm{e} 2}$ $=900 \mathrm{nH}$ are chosen to be the comparing set. It can be seen from the bode plot in Fig. 10 that the first zero frequency of the optimal set is $17.9 \mathrm{MHz}$ and the comparing set is $6.77 \mathrm{MHz}$. The ZCP frequency is very close to the optimal set path frequency but far away from the comparing set path frequency.

The main waveform of the optimal set and comparing set are shown in Fig.11. It can be observed from the high side current HV-I and low side current LV-I (Green and Blue curves) that there are nearly zero current oscillation going through the inductors with optimal set, but large high frequency current oscillation on comparing set. The measured oscillation frequency from the experiment is $4.6 \mathrm{MHz}$ which is slightly different from the calculated $6.77 \mathrm{MHz}$ path frequency, the bias mainly comes from the impedance measurement and parasitic derivation. It can be observed from the drain to source voltage of the high voltage side FET HV$V_{\text {ds }}$ (Red curve) that the optimal set are not achieving fully ZVS, thus introducing high frequency spike on top of the trapezoidal current. This might also cause the EMI problem. The partial ZVS condition with optimal set is because of the small inductance value assigned to the HV side inductor. Small HV side inductor stores less energy and requires longer dead time for charging and discharging the HV side FETs. In order to control variables, the dead time of two sets is 
manipulated equal but not enough for the optimal set for fully discharging the output capacitance $C_{\text {oss }}$ of the FET.

While keeping other conditions same, the efficiency measurement from the N4L precision power analyzer indicates that the optimal set is operating with $93.4 \%$ efficiency and the comparing set is operating with $89.5 \%$. Higher efficiency of the optimal set proves the lower loss in the magnetics components laterally. The magnetic thermal image of optimal set and comparing set are shown in Fig.12. The spot 1 is the HV side inductor and the optimal set temperature is $9{ }^{\circ} \mathrm{C}$ lower. The spot 2 is the Transformer and the optimal set temperature is $5.5^{\circ} \mathrm{C}$ lower. And the spot 3 is the LV side inductor and the optimal set temperature is $7.5^{\circ} \mathrm{C}$ lower. All magnetic components are operating with higher temperature in the comparing set validates that the HFO leads to extra loss. However, due to partial ZVS condition of the optimal set, the HV side FET are operating with slightly higher temperature than the comparing set achieving fully ZVS, which can be observed in Fig.13.

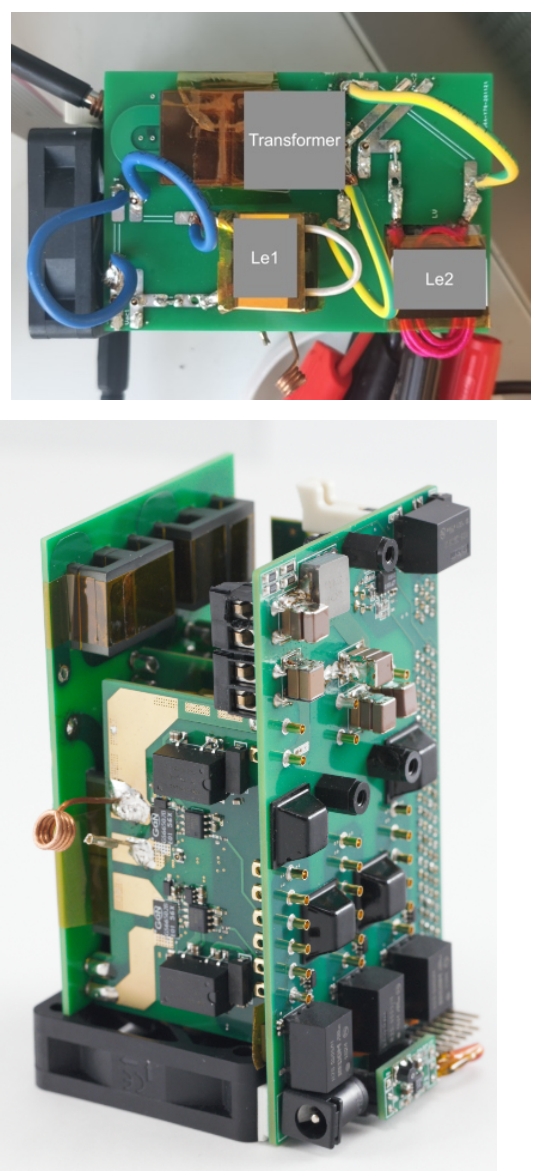

Fig.8. Experimental Prototype

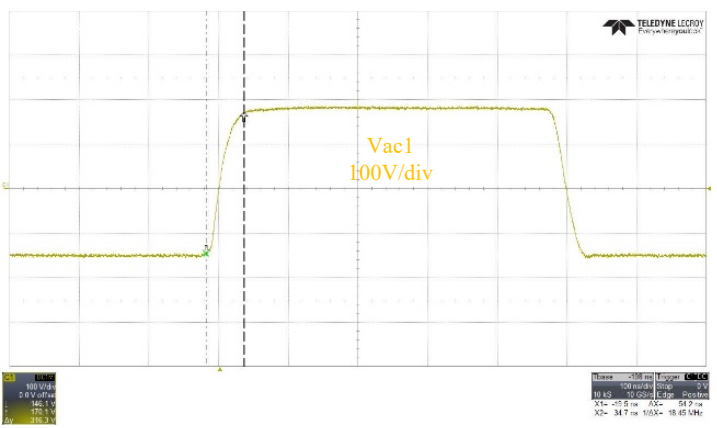

Fig.9. Waveforms of ac Link

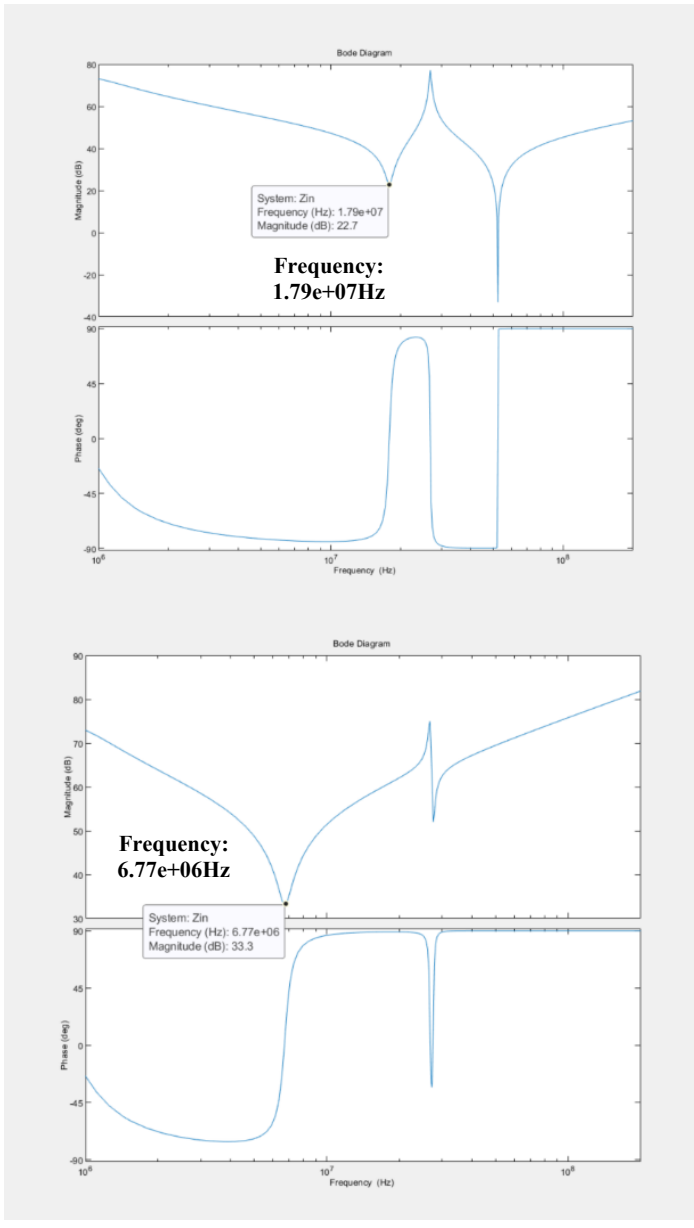

Fig.10. Bode Plot of the Input Impedance: Top-Optimal Set / Bottom-Comparing Set

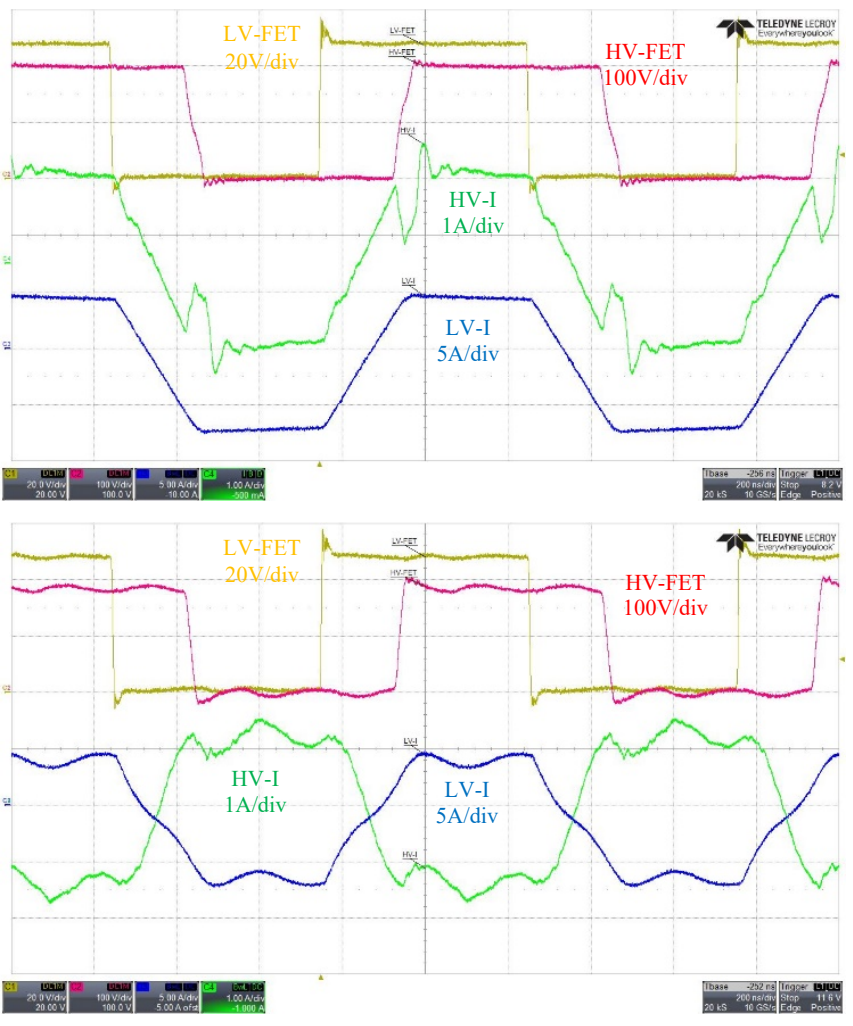

Fig.11. Main Waveforms: Top-Optimal Set / BottomComparing Set 


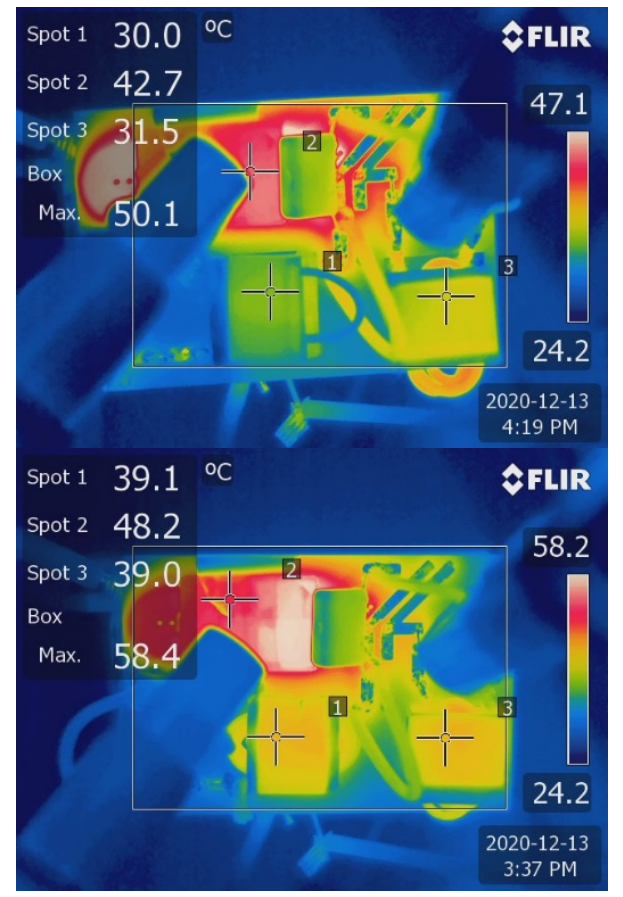

Fig.12. Magnetic Thermal Images: Top-Optimal Set / Bottom-Comparing Set
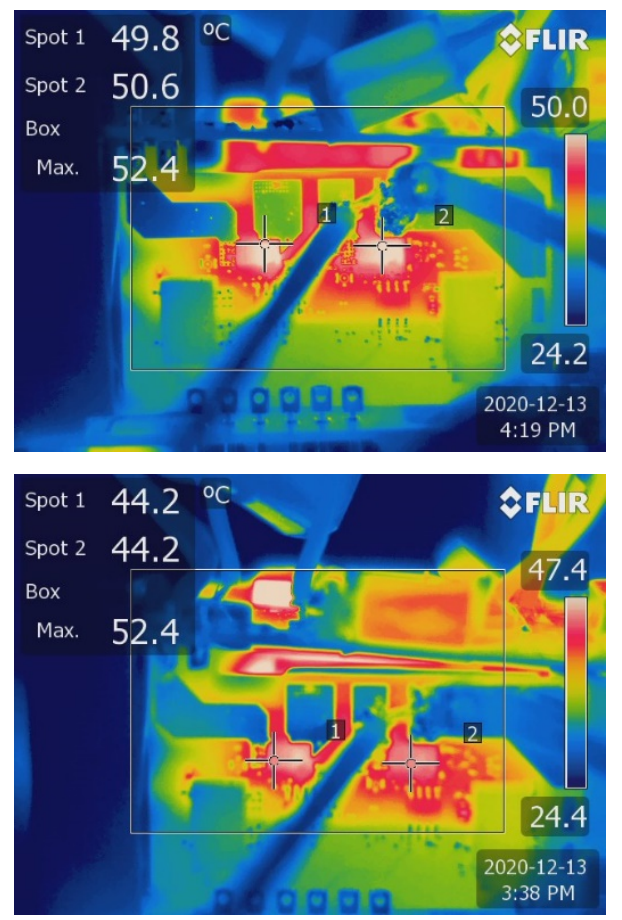

Fig.13. HV side FET Thermal Images: Top-Optimal Set / Bottom-Comparing Set

\section{CONCLUSION}

This paper proposed a splitting inductance tuning method to eliminate the high frequency oscillation problem in DAB converter. The impedance tuning approach is analyzed with the constructed prototype parameters. A $1 \mathrm{MHz}, 250 \mathrm{~W}$ DAB converter prototype is built and tested with an optimal set of inductors and a comparing set. The experimental comparison results validate the theoretical analysis and proves the feasibility of the proposed splitting inductance tuning method.

\section{REFERENCES}

[1] W. A. A. de Doncker and D. M. Divan, "A Three-phase Soft-Switched High-Power-Density dc/dc Converter for High-Power Applications," vol. 27, no. 1, 1991.

[2] Z. Zhang, J. Huang, and Y. Xiao, "GaN-based 1-MHz Partial Parallel Dual Active Bridge Converter with Integrated Magnetics," IEEE Transactions on Industrial Electronics, pp. 1-1, 2020, doi: 10.1109/TIE.2020.3007078.

[3] F. Krismer and J. W. Kolar, "Efficiency-optimized highcurrent dual active bridge converter for automotive applications," IEEE Transactions on Industrial Electronics, vol. 59, no. 7, pp. 2745-2760, Jul. 2012, doi: 10.1109/TIE.2011.2112312.

[4] W. Song, N. Hou, and M. Wu, "Virtual Direct Power Control Scheme of Dual Active Bridge DC-DC Converters for Fast Dynamic Response," IEEE Transactions on Power Electronics, vol. 33, no. 2, pp. 1750-1759, Feb. 2018, doi: 10.1109/TPEL.2017.2682982.

[5] C. Wang, M. Li, Z. Ouyang, and G. Wang, "Resonant Push-pull Converter with Flyback Regulator for MHz High Step-Up Power Conversion," IEEE Transactions on Industrial Electronics, pp. 1-1, 2020, doi: 10.1109/TIE.2020.2969109.

[6] Y. Wang, O. Lucia, Z. Zhang, S. Gao, Y. Guan, and D. Xu, "A Review of High Frequency Power Converters and Related Technologies," IEEE Open Journal of the Industrial Electronics Society, vol. 1, pp. 247-260, Sep. 2020, doi: 10.1109/ojies.2020.3023691.

[7] Y. Yan, H. Gui, and H. Bai, "Complete ZVS Analysis in Dual-Active-Bridge," IEEE Transactions on Power Electronics, vol. 8993, no. c, pp. 1-1, 2020, doi: 10.1109/tpel.2020.3011470.

[8] Z. Qin, Z. Shen, and F. Blaabjerg, "Modelling and analysis of the transformer current resonance in dual active bridge converters," in 2017 IEEE Energy Conversion Congress and Exposition (ECCE), Oct. 2017, pp. 4520-4524, doi: 10.1109/ECCE.2017.8096775.

[9] B. Cui, P. Xue, and X. Jiang, "Elimination of High Frequency Oscillation in Dual Active Bridge Converters by dv / dt Optimization," IEEE Access, vol. 7, pp. 5555455564, 2019, doi: 10.1109/ACCESS.2019.2910597.

[10] Y. Xiao, Z. Zhang, M. A. E. Andersen, and K. Sun, "Impact on ZVS Operation by Splitting Inductance to Both Sides of Transformer for 1-MHz GaN Based DAB Converter," IEEE Transactions on Power Electronics, pp. 1-1, 2020, doi: 10.1109/TPEL.2020.2988638. 